\title{
Desarrollo del mercado accionario y crecimiento económico en México: un examen mediante los enfoques ARDL y No causalidad
}

\author{
Stock market development and economic growth in Mexico: \\ An examination through ARDL and Non-causality approaches \\ Jesús Dacio Villarreal-Samaniego* \\ Instituto Tecnológico de Parral, México
}

Recibido el 23 de octubre de 2018; aceptado el 23 de septiembre de 2020

Disponible en Internet el: 23 de octubre de 2020

\section{Resumen}

Este trabajo de investigación examina la interacción entre el desarrollo del mercado de valores y el crecimiento económico en México. Las pruebas de raíz unitaria con cambios estructurales muestran que de todas las crisis registradas entre 1993 y el primer trimestre de 2018, la llamada Crisis del Tequila de mediados de la década de 1990 fue la más relevante ya que afectó a todas las variables examinadas. Al aplicar el modelo ARDL con pruebas de límites, el estudio encontró cointegración entre la dinámica de los indicadores económicos y varias variables del mercado de valores, pero no con el Índice de Precios y Cotizaciones. Los resultados de la prueba de no causalidad de Granger a través de la metodología Toda \& Yamamoto sugieren que la capitalización de mercado provocó el crecimiento económico en México e indican una causalidad bidireccional entre el crecimiento económico y el índice de capitalización de mercado.

Código JEL: G10, O10, O47

Palabras clave: Crecimiento económico; Mercado accionario; ARDL; Causalidad

\footnotetext{
*Autor para correspondencia

Correo electrónico: jesusdacio@yahoo.com (J.D. Villarreal Samaniego).

La revisión por pares es responsabilidad de la Universidad Nacional Autónoma de México. 


\title{
J. D. Villarreal Samaniego / Contaduría y Administración 66(3), 2021, 1-23 \\ http://dx.doi.org/10.22201/fca.24488410e.2021.2259
}

\begin{abstract}
This research paper examines the interaction between the development of the stock market and economic growth in Mexico. Unit root tests with structural changes show that of all crises registered between 1993 and the first quarter of 2018, the so-called Tequila Crisis of the mid-1990s was the most relevant since it affected all the variables examined. When applying the ARDL model with limits tests, the study found cointegration between the dynamics of economic indicators and several variables of the stock market, but not with the Price and Quotations Index. The results of the Granger non-causality test through the Toda \& Yamamoto methodology suggest that market capitalization caused economic growth in Mexico and indicate a two-way causality between economic growth and the market capitalization index.
\end{abstract}

JEL Code: $\mathrm{G} 10, \mathrm{O} 10, \mathrm{O} 47$

Keywords: Economic growth; Stock market; ARDL; Causality

\section{Introducción}

El crecimiento económico de un país, definido como el aumento en el valor de la producción en términos reales en un periodo de tiempo determinado, está muy relacionado con el bienestar de sus ciudadanos, así como con las perspectivas que tienen los productores, consumidores, inversionistas y demás agentes económicos para la toma de decisiones. Por estas razones el crecimiento económico, y las formas para impulsarlo, ha sido un tema de estudio constante en la ciencia económica y posiblemente sea el aspecto más examinado de esta disciplina.

En la literatura se ha argumentado que los mercados financieros cumplen una función importante al permitir que los recursos se muevan de aquellas unidades económicas que carecen de oportunidades de inversión productivas hacia las que sí las tienen (Levine \& Zervos, 1998; Mishkin \& Eakins, 2015). Es decir, un mercado financiero bien organizado permite dirigir los recursos hacia proyectos de inversión productivos y rentables lo que, en última instancia, promueve la actividad económica, contribuye a movilizar el ahorro de los hogares, favorece la diversificación del riesgo y facilita el intercambio de bienes y servicios (Caporale, Howells, \& Soliman, 2004).

Comenzando con el trabajo de Schumpeter (1911) y continuado con los de Goldsmith (1959) y McKinnon (1973), entre otros, múltiples estudios empíricos han explorado la relación causal entre el crecimiento económico y el desarrollo de los mercados financieros, aunque el debate respecto a sus hallazgos sigue en curso. En tanto que algunas investigaciones señalan una relación causal positiva entre el desarrollo de los mercados financieros y el crecimiento económico (Boubakari \& Jin, 2010; Caporale et al., 2004; King \& Levine, 1993; Pradhan, 
2018), otras concluyen que el desarrollo de los mercados financieros influye negativamente sobre el crecimiento económico (Bhatt, 1995; Lucas, 1988). Asimismo, aunque se argumenta que el sector financiero ayuda a movilizar ahorros para crear crecimiento en el sector real de la economía, también se afirma que los excedentes que genera el crecimiento económico provocan el desarrollo de los mercados financieros (Pan \& Mishra, 2018).

Sin embargo, considerando la complejidad y diversidad de los mercados financieros, varios estudios se han enfocado específicamente a examinar la relación causal entre el desarrollo de los mercados accionarios y el crecimiento económico (Adebayo, Awosusi, \& Eminer, 2020; Boubakari \& Jin, 2010; Lahura \& Vega, 2017; Levine \& Zervos, 1998; Pan \& Mishra, 2018). La presente investigación tiene como objetivo examinar la relación entre el crecimiento económico y el desarrollo del mercado accionario en México, país para el cual la literatura sobre el tema es relativamente escasa.

Específicamente, despúes de probar la presencia de raíz unitaria con cambios estructurales de acuerdo con la propuesta de Perron (1989) y de identificar los momentos de dichos cambios con el método de Bai \& Perron (2003) para generar las variables binarias correspondientes, el estudio aplica el modelo auto-regresivo de retardos distribuidos (ARDL) con prueba de límites (Pesaran, Shin, \& Smith, 2001) para examinar la dinámica a corto y largo plazos de las variables del mercado accionario y las de la economía real.

Además, dado el debate al respecto, se probó la existencia de no causalidad en el sentido de Granger con el método propuesto por Toda \& Yamamoto (1995), el cual permite determinar la relación y dirección de causalidad sin importar el orden de integración de las variables (Inusah, 2018). Tanto el modelo ARDL con prueba de límites como el de no causalidad de Toda y Yamamoto constituyen métodos potentes que, hasta donde se pudo determinar, no se han aplicado en los estudios previos realizados en México sobre el tema de esta investigación.

El resto del documento está organizado como sigue: la sección 2 presenta la revisión de la literatura, la parte 3 hace una descripción de los datos, la sección 4 explica la metodología empleada en la investigación, la parte 5 presenta los resultados empíricos, en tanto que la discusión y las conclusiones se presentan en la sección 6 .

\section{Revisión de la Literatura}

En la literatura se han propuesto diversos puntos de vista con respecto a la relación entre el desarrollo de los mercados financieros y el crecimiento económico (Blum, Federmair, Fink, \& Haiss, 2002; Graff, 2001). (1) El enfoque de que no existe relación entre tales variables. (2) La teoría de la anticipación a la oferta, en la que el desarrollo financiero se anticipa y determina el crecimiento económico (Cole \& Shaw, 1974; McKinnon, 1973). (3) La teoría del impulso de la demanda, que sostiene que el desarrollo financiero sigue al crecimiento 
económico (Robinson, 1952). (4) El enfoque de que existe una relación de interdependencia entre el sector real y el financiero (Patrick, 1966). (5) El punto de vista de que el desarrollo financiero obstaculiza el crecimiento económico (Bhatt, 1995; Lucas, 1988).

Diversos estudios señalan que hay una relación positiva entre la dinámica de la economía y la del mercado accionario. Pradhan (2018) examinó la relación entre el desarrollo del mercado accionario y el crecimiento económico en los países del G-20 entre 1980 y 2015. Las pruebas de causalidad en el sentido de Granger a través de modelos de vectores auto-regresivos (VAR) señalan que existe causalidad unidireccional y bidireccional entre el desarrollo del mercado accionario y el crecimiento económico per cápita. De forma similar, los resultados obtenidos por Caporale et al., (2004), en su análisis de siete países ${ }^{1}$ para el periodo 1977-1998, indican que un mercado accionario bien desarrollado promueve el crecimiento económico a largo plazo. Los autores concluyen también que los mercados accionarios que funcionan correctamente pueden propiciar el desarrollo económico, dando sustento al crecimiento económico a través de una acumulación más rápida de capital y ajustándolo por medio de una mejor asignación de los recursos.

Asimismo, los resultados de los modelos ARDL usados por Adebayo et al. (2020) para la economía nigeriana para el periodo 1989-2017, indican que la capitalización de mercado de las empresas listadas, el valor total de las acciones negociadas y la rotación del mercado tienen efectos positivos sobre el crecimiento económico, medido a través del PIB per cápita.

A pesar de la evidencia empírica que apoya la relación positiva entre el desarrollo del mercado accionario y el crecimiento económico, diversos estudios reportan hallazgos opuestos. En su análisis de nueve países candidatos a pertenecer a la unión europea ${ }^{2}$, Fink et al. (2009) no encontraron evidencia de que los mercados accionarios fueran un factor importante para el crecimiento económico de esas naciones en el periodo de 1996 a 2000. Por medio del enfoque ARDL con prueba de límites, Pan \& Mishra (2018) examinaron la relación entre el crecimiento económico, medido a través del índice de producción industrial como proxy del PIB, y el desarrollo del mercado accionario en China. El estudio, que consideró el periodo de enero de 1991 a noviembre de 2015, concluyó que, a largo plazo, el mercado A de Shanghái tuvo una relación muy pequeña, pero negativa, con la economía real. La misma investigación encontró que en el corto plazo no hubo relación alguna entre la economía real y el mercado accionario. Por otra parte, Men \& Li (2006) no encontraron una relación a largo plazo ni de causalidad en el sentido de Granger entre la economía china y los índices de las bolsas de Shanghái y de Shenzhen entre 1995 y 2005.

En los estudios realizados en varios países emergentes también se han reportado hallazgos conflictivos. Aali-Bujari, Venegas-Martínez, \& Pérez-Lechuga (2017) examinaron el impacto

\footnotetext{
${ }^{1}$ Argentina, Chile, Corea, Filipinas, Grecia, Malasia y Portugal.

${ }^{2}$ Bulgaria, Eslovaquia, Eslovenia, Hungría, Malta, Polonia, República Checa, Rumania y Turquía.
} 
del diferencial bancario y de la capitalización del mercado accionario sobre el crecimiento económico per cápita en las siete economías más importantes de América Latina ${ }^{3}$, variable que usaron como proxy del desarrollo económico. Esa investigación, que abarcó el periodo 1994-2012 y en la que se usó el método generalizado de momentos, concluyó que hay una relación positiva de la capitalización del mercado accionario con el ingreso per cápita y, por lo tanto, con el desarrollo económico de esos países de la región.

Tinoco et al. (2011) examinaron la influencia del desarrollo del mercado accionario, y del desarrollo financiero en general, sobre la tasa de crecimiento en cuatro países del Sudeste de Asia y tres de Latinoamérica ${ }^{4}$ entre 1980 y 2009 a través de regresión no paramétrica de panel. Los autores concluyeron que el desarrollo del mercado accionario ha tenido efectos negativos en el crecimiento económico de los países latinoamericanos, en tanto que tales efectos han sido favorables para los países del Sudeste de Asia. Lahura \& Vega (2017) aplicaron modelos VAR con restricciones a largo plazo para examinar la relación entre la actividad económica real y el desarrollo del mercado accionario en Perú en el periodo 1965-2013. Los resultados de dicha investigación indican que los shocks del mercado accionario tuvieron un efecto causal positivo a corto plazo sobre la dinámica del crecimiento del PIB real per cápita. Sin embargo, esto ocurrió solamente a partir de inicios de la década de 1990 y con un nivel bajo de impacto.

En el caso particular de México los estudios empíricos sobre la relación del desarrollo del mercado accionario y el crecimiento de la economía también reportan resultados mixtos. Kassimatis \& Spyrou (2001) investigaron la relación entre el desarrollo del sector bancario, el mercado accionario y el desarrollo económico de cinco de las economías emergentes más importantes ${ }^{5}$ entre 1977 y 1997, a través de un método multivariado de series temporales. Dicha investigación concluyó que el mercado accionario juega un papel en el desarrollo económico en México. Más específicamente, los resultados del estudio indican que el mercado accionario mexicano es exógeno, según el modelo de Boyd \& Smith (1996), lo que los autores atribuyen al alto nivel de apertura económica del país.

Ron Delgado (2001) examinó la relación a largo plazo y causal entre la actividad del mercado de acciones y el crecimiento económico en México entre 1980 y 2001, empleando modelos VAR, así como el método de no causalidad de Granger. Los resultados de esa investigación mostraron que el efecto del indicador del desarrollo del mercado de valores fue significativo para el PIB entre 1987 y 2001 . Así mismo, el estudio concluyó que el Índice de Precios y Cotizaciones (IPC) de la Bolsa Mexicana de Valores y el valor de la producción industrial tuvieron una relación de equilibrio a largo plazo, y que el IPC causa en el sentido de Granger al valor de la producción industrial.

\footnotetext{
${ }^{2}$ Argentina, Brasil, Chile, Colombia, México, Perú y Venezuela.

${ }^{4}$ Los países del Sudeste de Asia fueron Filipinas, Indonesia, Malasia y Tailandia y los de Latinoamérica fueron Chile, México y Venezuela.

${ }^{5}$ Chile, Corea del Sur, India, México y Taiwán.
} 
La investigación de Castillo-Ponce, Rodriguez-Espinosa, \& Gaytan-Alfaro (2015) examinó la relación entre el crecimiento económico, medido a través de la dinámica del PIB y de la producción industrial, y diversos indicadores del mercado de valores en México entre 1993 y el primer trimestre de 2011. Al aplicar la prueba de cointegración de Johansen (1991) y de ciclo común de Vahid \& Engle (1993), los autores encontraron una asociación positiva a largo plazo entre las variables del mercado accionario y el crecimiento económico, por lo que concluyeron que el desarrollo del mercado accionario propicia el crecimiento económico en México.

Los resultados de esas tres investigaciones, sin embargo, contrastan con los hallazgos reportados por Tinoco et al. (2011), quienes concluyeron que el desarrollo del mercado accionario mexicano tiene efectos negativos sobre el crecimiento económico del país.

\section{Descripción de los Datos}

El periodo del estudio fue del primer trimestre de 1993 al segundo trimestre de 2018 para los datos con esa periodicidad, y de enero de 1993 a febrero de 2018 para los datos con frecuencia mensual. En ese lapso se presentaron diversos eventos económicos y financieros importantes tanto en el entorno nacional, como la Crisis del Tequila, como en el internacional, como la denominada Gran Recesión de 2008-2009, que sugieren la posible presencia de cambios estructurales en las variables.

En su investigación, Beck, Demirgüç-Kunt, \& Levine (2010) hacen referencia a las variables asociadas con el desarrollo del mercado accionario que han sido más frecuentemente usadas en la literatura. Entre tales variables están los indicadores del tamaño del mercado, como la razón de capitalización del mercado accionario como porcentaje del PIB; los de actividad, como la razón del valor negociado total del mercado accionario como porcentaje del PIB; y los de eficiencia, como la rotación del mercado accionario, calculada como el volumen de operaciones por periodo entre la capitalización total del mercado.

Cuche \& Hess (1999) afirman que, aunque indudablemente el PIB es un indicador clave del crecimiento de la economía, es conveniente usar una variable relacionada con éste, pero con una mayor frecuencia de medición, con el fin de evitar la desventaja de las observaciones trimestrales. En su artículo seminal sobre vectores auto-regresivos, Sims (1992) usó el índice de producción industrial como proxy del PIB. Este indicador del crecimiento económico también ha sido usado en diversas investigaciones posteriores (véase, por ejemplo, Castillo-Ponce et al., 2015; Kajurová, 2017; Pan \& Mishra, 2018; Paramati \& Gupta, 2013; Ron Delgado, 2001). Así pues, en este estudio se emplearon los datos trimestrales del producto interno bruto en términos reales a precios de 2013, así como los datos mensuales del Índice de Producción Industrial (PI) con base 100 al año 2015 como variables relacionadas con el crecimiento económico. 
Asimismo, los indicadores del desarrollo del mercado accionario se seleccionaron de acuerdo con lo que se ha planteado previamente en la literatura (véase, por ejemplo, Adebayo et al., 2020; Beck et al., 2010; Ghimire \& Giorgioni, 2013; Ho \& Iyke, 2017; Pradhan, 2018). Diversas investigaciones consideran a los índices accionarios como proxy del tamaño del mercado, por lo que aquí se incluye al IPC, el principal índice accionario de la Bolsa Mexicana de Valores, como se ha hecho en estudios previos (Castillo-Ponce et al., 2015; Ron Delgado, 2001). No obstante, se ha señalado que los índices accionarios no necesariamente son un buen proxy del tamaño del mercado debido al proceso de selección de los componentes, en el que intervienen comités. Además, dado el método usado para su cálculo, el valor de los índices está fuertemente influenciado por los movimientos en los precios de las acciones de las empresas más grandes, que frecuentemente son multinacionales (Pan \& Mishra, 2018).

Esta investigación también considera otras variables relacionadas con el tamaño y otros aspectos del mercado accionario mexicano. La capitalización del mercado $(K)$ es el número total de acciones en circulación de cada emisor en el mercado multiplicado por el precio respectivo, en tanto que el volumen de operaciones $(O)$ es el valor monetario promedio de las acciones negociadas en un periodo determinado. Estas variables, que se miden en términos reales, se relacionan con el tamaño y la liquidez del mercado, respectivamente. Además, se consideran estos mismos indicadores en términos relativos con respecto a las variables del crecimiento económico. Las razones de capitalización del mercado $(K / P I B$ y $K / P I)$ y de volumen negociado $(O / P I B$ y $O / P I)$, se relacionan con el tamaño y la actividad relativa del mercado, respectivamente. La rotación del mercado, es decir, el volumen negociado con respecto a la capitalización $(O / K)$, se usa como medida de liquidez.

Los datos trimestrales del PIB se obtuvieron del Instituto Nacional de Estadística y Geografía (INEGI), en tanto que los del PI con frecuencia mensual se consiguieron en la base de datos de la Organización para la Cooperación y el Desarrollo Económicos (OCDE). Las observaciones de las variables relacionadas con el mercado de valores se obtuvieron de la base de datos publicada por el Banco de México.

\section{Aspectos Metodológicos}

Para determinar la presencia de raíz unitaria con cambios estructurales en las series, se aplicó la prueba de minimización del estadístico $t$ de Dickey-Fuller. Luego se identificaron los momentos en los que se presentaron tales cambios mediante el modelo de $L$ vs. $L+1$ cambios secuencialmente determinados de Bai \& Perron (2003), considerando la cantidad de retardos sugeridos por el criterio de información de Schwarz (SIC). 
Tabla 1

Pruebas de raíz unitaria con cambios estructurales de las variables en niveles

\begin{tabular}{|c|c|c|c|c|c|c|c|c|c|}
\hline \multicolumn{10}{|c|}{ Panel A: Frecuencia Mensual (M) } \\
\hline \multicolumn{5}{|c|}{ Niveles } & \multicolumn{5}{|c|}{ Primeras Diferencias } \\
\hline (1) & (2) & (3) & (4) & (5) & (6) & (7) & (8) & (9) & (10) \\
\hline Variable & Estadísitico $t$ & Valor- $p$ & $\begin{array}{c}\text { ¿Raíz } \\
\text { Unitaria? }\end{array}$ & $\begin{array}{c}\text { Fecha del } \\
\text { Cambio }\end{array}$ & Estadísitico $t$ & Valor- $p$ & $\begin{array}{c}\text { ¿Raíz } \\
\text { Unitaria? }\end{array}$ & $\begin{array}{l}\text { Fecha del } \\
\text { Cambio }\end{array}$ & $I(d)$ \\
\hline PI & -4.0155 & 0.152 & $\mathrm{Si}$ & -- & -18.9504 & $<0.01$ & No* & 1994M02 & $I(1)$ \\
\hline IPC & -2.5244 & 0.896 & $\mathrm{Si}$ & --- & -18.4688 & $<0.01$ & No* & 2008M10 & $I(1)$ \\
\hline $\mathrm{K}$ & -2.4582 & 0.913 & $\mathrm{Si}$ & --- & -18.0945 & $<0.01$ & No* & 2008M10 & $I(1)$ \\
\hline $\mathrm{K} / \mathrm{PI}$ & -2.4625 & 0.912 & $\mathrm{Si}$ & --- & -24.9541 & $<0.01$ & No* & $2008 \mathrm{M} 10$ & $I(1)$ \\
\hline $\mathrm{O}$ & -4.5441 & 0.038 & $\mathrm{No}^{* *}$ & 2009M01 & --- & --- & --- & --- & $I(0)$ \\
\hline $\mathrm{O} / \mathrm{PI}$ & -4.5866 & 0.034 & No** & 1994M05 & --- & --- & --- & --- & $I(0)$ \\
\hline $\mathrm{O} / \mathrm{K}$ & -21.6415 & $<0.01$ & No* & 2001M08 & --- & --- & -- & --- & $I(0)$ \\
\hline \multicolumn{10}{|c|}{ Panel B: Frecuencia Trimestral (Q) } \\
\hline \multicolumn{5}{|c|}{ Niveles } & \multicolumn{4}{|c|}{ Primeras Diferencias } & \\
\hline (1) & (2) & (3) & (4) & (5) & (6) & (7) & $(8)$ & (9) & (10) \\
\hline Variable & Estadísitico $t$ & Valor- $p$ & $\begin{array}{c}\text { ¿Raíz } \\
\text { Unitaria? }\end{array}$ & $\begin{array}{c}\text { Fecha del } \\
\text { Cambio }\end{array}$ & Estadísitico $t$ & Valor- $p$ & $\begin{array}{c}\text { ¿Raíz } \\
\text { Unitaria? }\end{array}$ & $\begin{array}{c}\text { Fecha del } \\
\text { Cambio }\end{array}$ & $I(d)$ \\
\hline PIB & -0.1074 & $>0.99$ & $\mathrm{Si}$ & --- & -18.2901 & $<0.01$ & No* & 1995Q1 & $I(1)$ \\
\hline IPC & -2.5062 & 0.902 & $\mathrm{Si}$ & --- & -8.7800 & $<0.01$ & No* & 2008Q3 & $I(1)$ \\
\hline $\mathrm{K}$ & -2.3727 & 0.933 & $\mathrm{Si}$ & -- & -8.8692 & $<0.01$ & No* & 2008Q3 & $I(1)$ \\
\hline $\mathrm{K} / \mathrm{PIB}$ & -3.1389 & 0.604 & $\mathrm{Si}$ & --- & -10.0745 & $<0.01$ & No* & 1995Q1 & $I(1)$ \\
\hline $\mathrm{O}$ & -7.1681 & $<0.01$ & No* & 2009Q2 & --- & --- & --- & --- & $I(0)$ \\
\hline $\mathrm{O} / \mathrm{PIB}$ & -6.0187 & $<0.01$ & No* & 2009Q2 & --- & --- & --- & --- & $I(0)$ \\
\hline $\mathrm{O} / \mathrm{K}$ & -8.6749 & $<0.01$ & No* & 2013Q2 & --- & -- & -- & -- & $I(0)$ \\
\hline
\end{tabular}

* Significativo al 1\% * $\quad * *$ Significativo al 5\% *** Significativo al 10\%

Fuente: Elaboración propia.

Los resultados de las pruebas de raíz unitaria se muestran en la Tabla 1. En el panel A se encuentran las variables medidas con una periodicidad mensual y en el B las que se observan con una periodicidad trimestral. Tales resultados indican que, independientemente de la frecuencia de medición, las series que involucran de alguna manera al volumen de operaciones o a las razones de mercado relacionadas con esa variable resultaron estacionarias en niveles, mientras que el resto de las variables fueron estacionarias en primeras diferencias. Además, estos resultados evidencian que los cambios estructurales identificados coinciden, en efecto, con las crisis ocurridas entre mediados de la década de 1990 y 2013. 
Los resultados de las pruebas de raíz unitaria de los logaritmos del $P I, P I B, K$ y $O$ se muestran en la Tabla 2. La mayoría de los cambios estructurales en los logaritmos de prácticamente todas las variables coinciden con la Crisis del Tequila originada en México, lo que es evidencia de la considerable influencia que tuvo ese evento. También se observa que algunas de las variables resultaron estacionarias en niveles, mientras que otras lo son en primeras diferencias.

Tabla 2

Pruebas de raíz unitaria con cambios estructurales de los logaritmos de las variables

\begin{tabular}{|c|c|c|c|c|c|c|c|c|c|}
\hline \multicolumn{10}{|c|}{ Panel A: Frecuencia Mensual (M) } \\
\hline \multicolumn{5}{|c|}{ Niveles } & \multicolumn{5}{|c|}{ Primeras Diferencias } \\
\hline (1) & (2) & (3) & (4) & (5) & (6) & (7) & (8) & (9) & (10) \\
\hline Variable & Estadísitico $t$ & Valor- $p$ & $\begin{array}{c}\text { ¿Raíz } \\
\text { Unitaria? }\end{array}$ & $\begin{array}{c}\text { Fecha del } \\
\text { Cambio }\end{array}$ & Estadísitico $t$ & Valor- $p$ & $\begin{array}{c}\text { ¿Raíz } \\
\text { Unitaria? }\end{array}$ & $\begin{array}{c}\text { Fecha del } \\
\text { Cambio }\end{array}$ & $I(d)$ \\
\hline $\ln (\mathrm{PI})$ & -4.3162 & 0.072 & No*** & $1995 \mathrm{M} 10$ & --- & -- & --- & --- & $I(0)$ \\
\hline $\ln ($ IPC) & -3.5081 & 0.383 & $\mathrm{Si}$ & -- & -18.7658 & $<0.01$ & No* & 1995M02 & $I(1)$ \\
\hline $\ln (\mathrm{K})$ & -3.5444 & 0.363 & $\mathrm{Si}$ & --- & -16.8508 & $<0.01$ & No* & 1998M08 & $I(1)$ \\
\hline $\ln (\mathrm{O})$ & -4.8538 & 0.015 & $\mathrm{No}^{* *}$ & $2005 \mathrm{M} 07$ & --- & --- & --- & --- & $I(0)$ \\
\hline \multicolumn{10}{|c|}{ Panel B: Frecuencia Trimestral (Q) } \\
\hline & \multicolumn{4}{|c|}{ Niveles } & \multicolumn{4}{|c|}{ Primeras Diferencias } & \\
\hline (1) & (2) & (3) & (4) & $(5)$ & (6) & (7) & $(8)$ & (9) & (10) \\
\hline Variable & Estadísitico $t$ & Valor- $p$ & $\begin{array}{c}\text { ¿Raíz } \\
\text { Unitaria? }\end{array}$ & $\begin{array}{c}\text { Fecha del } \\
\text { Cambio }\end{array}$ & Estadísitico $t$ & Valor- $p$ & $\begin{array}{c}\text { ¿Raíz } \\
\text { Unitaria? }\end{array}$ & $\begin{array}{c}\text { Fecha del } \\
\text { Cambio }\end{array}$ & $I(d)$ \\
\hline $\ln (\mathrm{PIB})$ & -5.4246 & $<0.01$ & No* & 1994Q3 & -- & -- & --- & --- & $I(0)$ \\
\hline $\ln (\mathrm{IPC})$ & -3.5670 & 0.349 & $\mathrm{Si}$ & --- & -9.6071 & $<0.01$ & No* & 1995Q1 & $I(1)$ \\
\hline $\ln (\mathrm{K})$ & -3.5842 & 0.340 & $\mathrm{Si}$ & --- & -9.4632 & $<0.01$ & No* & 1995Q1 & $I(1)$ \\
\hline $\ln (\mathrm{O})$ & -4.1369 & 0.115 & $\mathrm{Si}$ & -- & -15.1583 & $<0.01$ & No* & 1996Q3 & $I(1)$ \\
\hline
\end{tabular}

* Significativo al 1\% * * Significativo al 5\% * ** Significativo al $10 \%$

Fuente: Elaboración propia.

En la literatura sobre la relación entre el crecimiento económico y el desarrollo del mercado accionario se han usado diversas pruebas de cointegración como la de máxima verosimilitud (Johansen, 1988), la de residuales (Engle y Granger, 1987), la de cambios de régimen (Hatemi-J, 2008) y el modelo auto-regresivo de retardos distribuidos (ARDL) con prueba de límites propuesto (Pesaran et al., 2001). Sin embargo, éste último ofrece algunas características que son ventajosas sobre otras pruebas de cointegración. El método ARDL simplifica el análisis de la relación de la respuesta y las variables de entrada a través de regresiones mínimos cuadrados ordinarios, además de que toma en cuenta un número suficiente de retardos para considerar 
el proceso generador de datos en un contexto que va de lo general a lo específico (Hoque \& Yakob, 2017; Laurencenson \& Chai, 2003). Sin embargo, sobre la base de los resultados de las pruebas de raíz unitaria de las Tablas 1 y 2, para esta investigación la principal ventaja que ofrece el método ARDL es que es aplicable a variables con diferente orden de integración, en tanto éste no sea mayor a I(1) (Menegaki, 2019; Shestha \& Chowdury, 2005).

La especificación ARDL general utilizada en el estudio es el siguiente modelo no restringido de corrección del error (ECM) que incluye variables binarias para los cambios estructurales:

$$
\Delta Y_{n, t}=\alpha_{0}+\sum_{i=1}^{p} \alpha_{j} \Delta Y_{n, i}+\sum_{i=0}^{q_{1}} \beta_{j} \Delta X_{m, i}+\delta_{Y} Y_{n, t-1}+\delta_{X} X_{m, t-1}+\delta_{D} D_{k, t}+\varepsilon_{t}
$$

En la ecuación (1) $\alpha_{0}$ es la constante, $Y$ es el valor de la $n$-ésima variable económica de respuesta $(P I B$ o $P I), X_{m}$ es la $m$-ésima variable del mercado accionario, $D_{k}$ es una variable binaria que toma el valor de 1 en el $k$-ésimo régimen y de cero en cualquier otro caso. Los coeficientes $\alpha_{j}$ y $\beta_{j}$ son las relaciones a corto plazo, los coeficientes $\delta_{i}$ representan los parámetros a largo plazo y $\varepsilon_{t}$ es el error aleatorio, i.i.d. Cuando la prueba de límites rechazó la hipótesis nula de que no hay una relación de cointegración, se utilizó el siguiente ECM dentro de la estructura de modelación ARDL:

$$
\Delta Y_{n, t}=\alpha_{0}+\sum_{i=1}^{p} \alpha_{j} \Delta Y_{n, i}+\sum_{i=0}^{q_{1}} \beta_{j} \Delta X_{m, i}+\varphi E C T_{t-1}+\delta_{D} D_{k, t}+\varepsilon_{t}
$$

En la ecuación (2), la velocidad de ajuste, $\phi$, del término de corrección del error, $E C T_{t-1}$, debe ser negativa y estadísticamente significativa para validar la relación a largo plazo entre las variables. El número de retardos de los modelos se determinó mediante el criterio de información de Akaike (AIC). En la mayoría de los casos se utilizaron errores estándar consistentes con la heterocedasticidad y la autocorrelación (HAC) (Newey \& West, 1987), excepto para el de la relación entre el $P I B$ y el $I P C$ y el de la relación entre el $P I B$ y la razón $K / P I B$, en donde no se pudo rechazar la presencia de homocedasticidad.

Siguiendo la propuesta de Pesaran y Pesaran (1997) se aplicaron las pruebas de control de sumas acumuladas de los residuos recursivos (CUSUM) y las de sumas acumuladas de los cuadrados de los residuos recursivos (CUSUMSQ) para evaluar la consistencia de los 
parámetros (Brown, Durbin y Evans, 1975). Todos los modelos resultaron estables según ambas pruebas a un nivel de significancia del $5 \%$.

Con el fin de probar la no causalidad de Granger, se emplearon procesos de vectores autorregresivos (VAR) según el procedimiento propuesto por Toda \& Yamamoto (1995). Una vez que se determinó el tamaño máximo de retardos, $r$, se estimó el $\left(r+d_{\max }\right)$-iésimo orden del VAR, siendo $d_{\max }$ el orden máximo de integración que se asume ocurre en el proceso. Luego se aplicó la prueba de Wald considerando los coeficientes de los retardos $\left(r+d_{\max }\right)$ adicionales como variables exógenas. El modelo VAR general empleado para probar la no causalidad de Granger fue:

$$
\begin{gathered}
Y_{n, t}=\alpha_{0}+\sum_{i=1}^{p} \alpha_{i} Y_{n, t-i}+\sum_{i=p+1}^{d \max } \alpha_{i} Y_{n, t-i}+\sum_{j=1}^{p} \beta_{j} X_{m, t-j}+\sum_{j=p+1}^{d m a x} \beta_{j} X_{m, t-j}+v_{t} \\
X_{m, t}=\gamma_{0}+\sum_{i=1}^{p} \gamma_{i} X_{m, t-i}+\sum_{i=p+1}^{d \max } \gamma_{i} X_{m, t-i}+\sum_{j=1}^{p} \delta_{j} Y_{n, t-j}+\sum_{j=p+1}^{d \max } \delta_{j} Y_{n, t-j}+v_{t}
\end{gathered}
$$

En las ecuaciones (3) y (4), $\alpha_{i}, \beta_{j}, \gamma_{i}$ y $\delta_{j}$ son los parámetros desconocidos de los modelos, mientras que $v$ y $v$ son las perturbaciones i.i.d. Para la ecuación (3) $\mathrm{H}_{0}: \beta_{1}=\beta_{2}=\ldots=\beta_{p}=0$ $\mathrm{y}_{1}: \beta_{1} \neq \beta_{2} \neq \ldots \neq \beta_{p} \neq 0$, y para la (4) $\mathrm{H}_{0}: \delta_{1}=\delta_{2}=\ldots=\delta_{p}=0 \mathrm{y} \mathrm{H}_{1}: \delta_{1} \neq \delta_{2} \neq \ldots \neq \delta_{p} \neq 0$.

\section{Resultados}

\section{Análisis Gráfico}

Como una primera aproximación al análisis de las series temporales consideradas en el estudio, la Figura 1 presenta una gráfica con las variables del crecimiento económico, así como las del mercado accionario con periodicidad trimestral. El panel A muestra la dinámica del $I P C$ y del $P I B$ en términos reales, y se observa una relación estrecha en el comportamiento de ambas variables.

En el panel B se observa el comportamiento del IPC y de la capitalización del mercado, mientras que en el panel C se observa la dinámica del IPC y del volumen de operaciones. En ambos casos se advierte una relación considerable entre las variables representativas de la

\footnotetext{
${ }^{6}$ En algunos modelos se presentaron periodos breves de inestabilidad según la prueba CUSUMSQ mismos que tuvieron una duración de 1 a 14 periodos para los datos con frecuencia mensual y de un periodo para los datos con frecuencia trimestral, aunque con un retorno a la estabilidad en todos los casos.
} 
actividad del mercado accionario y también reflejan las crisis que se presentaron en el periodo bajo estudio. Se hacen evidentes los considerables incrementos en el volumen de operaciones relacionados con la Gran Recesión de 2008 y, de forma muy notoria, con la Rabieta de Disminución Gradual de 2013, aunque tales aumentos fueron de corta duración.
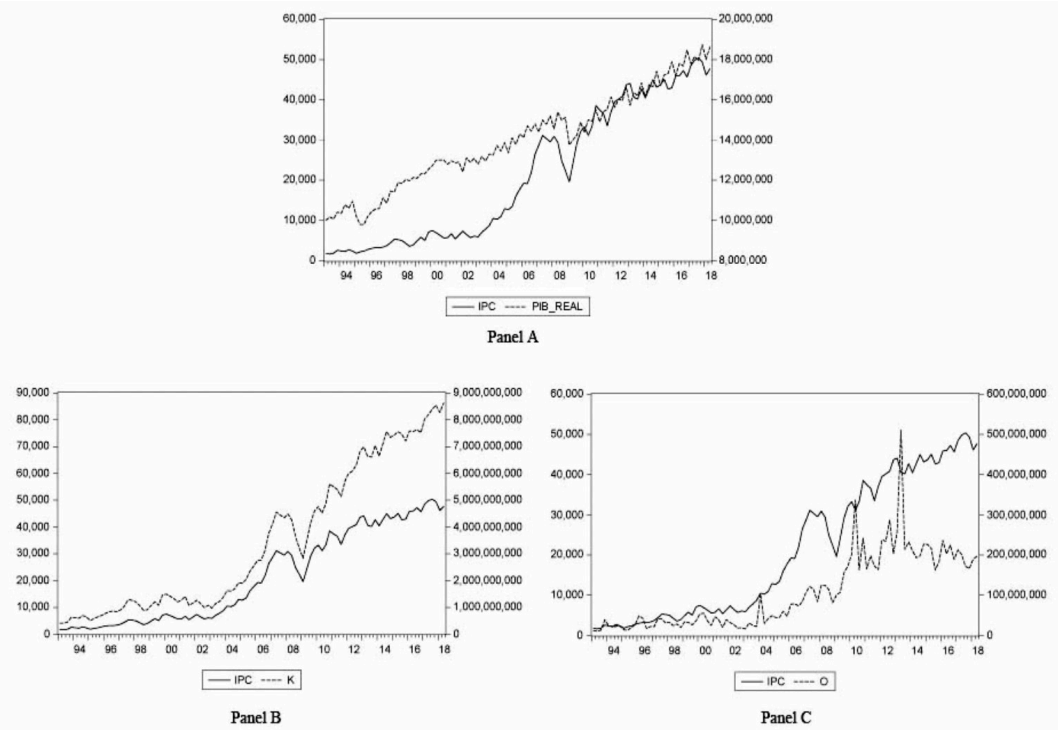

Figura 1. Indicadores del mercado bursátil y del crecimiento económico (1993 - 2018).

Fuente: Elaboración propia con datos del INEGI y del Banco de México.

Especificación de los Momentos de los Cambios Estructurales

Para determinar los momentos en los que ocurrieron los cambios estructurales se plantearon modelos lineales bivariados con la siguiente forma:

$$
Y_{n, t}=\alpha+\beta X_{m, t}+\varepsilon_{t}
$$

En estas especificaciones $Y_{n}$ y $X_{m}$ son, respectivamente, las variables relacionadas con el crecimiento económico y con el mercado como se definieron anteriormente, $\alpha$ y $\beta$ son parámetros desconocidos y $\varepsilon_{t}$ es la perturbación, como se define usualmente. 
Tabla 3

Fechas de los cambios estructurales en las ecuaciones bivariadas

\begin{tabular}{|c|c|c|c|c|c|c|c|c|c|}
\hline \multicolumn{5}{|c|}{ Panel A. Variable de Respuesta: PI } & \multicolumn{5}{|c|}{ Panel B. Variable de Respuesta: $\ln (\mathrm{IMAI})$} \\
\hline \multicolumn{5}{|c|}{ Frecuencia Mensual (M) } & \multicolumn{5}{|c|}{ Frecuencia Mensual (M) } \\
\hline Variable & \multicolumn{4}{|c|}{ Fechas de los Cambios } & Variable & \multicolumn{4}{|c|}{ Fechas de los Cambios } \\
\hline IPC & 1998M05 & 2005M05 & 2009M03 & 2014M02 & $\ln (\mathrm{IPC})$ & 1998M02 & 2009M04 & 2014M02 & --- \\
\hline $\mathrm{K}$ & 1998M07 & 2002M07 & $2006 \mathrm{M} 10$ & 2014M04 & $\ln (\mathrm{K})$ & 1998M02 & 2002M07 & 2009M03 & 2014M04 \\
\hline $\mathrm{O}$ & 1998M01 & 2004M03 & 2008M03 & $2011 \mathrm{M} 12$ & $\ln (\mathrm{O})$ & 1997M11 & 2003M12 & 2008M03 & 2011M12 \\
\hline $\mathrm{K} / \mathrm{PI}$ & 1998M02 & 2002M07 & 2006 M11 & 2014M02 & & & & & \\
\hline $\mathrm{O} / \mathrm{K}$ & 1997M04 & 2004M02 & 2011M07 & --- & & & & & \\
\hline $\mathrm{O} / \mathrm{PI}$ & 1997M11 & 2004M03 & 2008M03 & $2011 \mathrm{M} 12$ & & & & & \\
\hline \multicolumn{5}{|c|}{ Panel C. Variable de Respuesta: PIB } & \multicolumn{5}{|c|}{ Panel D. Variable de Respuesta: $\ln (\mathrm{PIB})$} \\
\hline \multicolumn{5}{|c|}{ Frecuencia Trimestral (Q) } & \multicolumn{5}{|c|}{ Frecuencia Trimestral (Q) } \\
\hline $\begin{array}{c}\text { Variable } \\
\text { Independiente }\end{array}$ & \multicolumn{4}{|c|}{ Fechas de los Cambios } & $\begin{array}{c}\text { Variable } \\
\text { Independiente }\end{array}$ & \multicolumn{4}{|c|}{ Fechas de los Cambios } \\
\hline IPC & 1998Q2 & $2002 \mathrm{Q} 2$ & 2009Q2 & 2014Q4 & $\ln (\mathrm{IPC})$ & 1998Q2 & 2002Q2 & 2008Q2 & 2013Q2 \\
\hline $\mathrm{K}$ & 1998Q2 & 2002Q2 & 2011Q1 & 2014Q4 & $\ln (\mathrm{K})$ & 1998Q2 & 2002Q2 & 2010Q4 & 2014Q3 \\
\hline $\mathrm{O}$ & 1997Q4 & 2001Q3 & 2005Q2 & 2013Q3 & $\ln (\mathrm{O})$ & 1999Q3 & 2004Q2 & 2013Q3 & --- \\
\hline $\mathrm{K} / \mathrm{PIB}$ & 2000Q1 & 2003Q2 & 2012Q4 & --- & & & & & \\
\hline $\mathrm{O} / \mathrm{K}$ & 1997Q4 & 2002Q2 & 2006Q2 & 2013Q3 & & & & & \\
\hline $\mathrm{O} / \mathrm{PIB}$ & 1997Q4 & 2001Q3 & 2005Q4 & 2013Q3 & & & & & \\
\hline
\end{tabular}

Método: L+1 vs. L cambios estructurales determinados secuencialmente (Bai - Perron, 2003).

Fuente: Elaboración propia.

Los momentos de los cambios de régimen establecieron con el método de $L+1$ vs. $L$ cambios estructurales determinados secuencialmente (Bai \& Perron, 2003), cuyos resultados se usaron para generar las variables binarias correspondientes. La Tabla 3 muestra los hallazgos de dichas pruebas, tanto para los datos mensuales, en los paneles A y B, como para los datos trimestrales que se muestran en los paneles $\mathrm{C}$ y D.

\section{Prueba de Límites}

Los resultados de la prueba de límites de los modelos ARDL aparecen en las Tablas 4 y 5 . En los paneles A y B de la Tabla 4 se pueden observar, respectivamente, los resultados de los modelos que incluyeron al PI y al PIB como variable de respuesta. Asimismo, en los paneles A y B de la Tabla 5 se muestran los resultados correspondientes a los logaritmos de estas dos variables, también respectivamente. 
Tabla 4

Resultados de las pruebas de límites: Variables en niveles

Panel A. Variable de Respuesta: PI

Datos Mensuales

\begin{tabular}{|c|c|c|c|c|c|}
\hline & \multicolumn{3}{|c|}{ Resultados ARDL } & \multirow[b]{2}{*}{ (5) } & \multirow[b]{2}{*}{ (6) } \\
\hline (1) & (2) & (3) & (4) & & \\
\hline $\begin{array}{c}\text { Variable } \\
\text { Independiente }\end{array}$ & Caso & Estadístico F & Decisión $\mathrm{H}_{0}$ & $\begin{array}{c}\text { Coeficiente de } \\
\text { Corrección del Error } \\
\text { (EC) }\end{array}$ & $\begin{array}{c}\text { Valor p Coeficiente } \\
\text { a Largo Plazo }\end{array}$ \\
\hline IPC & III & 3.86 & Aceptar $\mathrm{H}_{0}$ & --- & --- \\
\hline $\mathrm{K} / \mathrm{PI}$ & III & 9.63 & Rechazar $\mathrm{H}_{0} *$ & $-0.0838^{*}$ & 0.00 \\
\hline $\mathrm{O} / \mathrm{K}$ & IV & 6.67 & Rechazar $\mathrm{H}_{0}{ }^{* *}$ & $-0.0975^{*}$ & 0.52 \\
\hline $\mathrm{O} / \mathrm{PI}$ & IV & 6.16 & Rechazar $\mathrm{H}_{0} * *$ & $-0.0926^{*}$ & 0.21 \\
\hline K & III & 7.34 & Rechazar $\mathrm{H}_{0}{ }^{* *}$ & $-0.0674 *$ & 0.00 \\
\hline $\mathrm{O}$ & IV & 6.73 & Rechazar $\mathrm{H}_{0} * *$ & $-0.0960 *$ & 0.15 \\
\hline \multicolumn{6}{|c|}{ Panel B. Variable de Respuesta: PIB } \\
\hline \multicolumn{6}{|c|}{ Datos Trimestrales } \\
\hline & \multicolumn{3}{|c|}{ Resultados ARDL } & & \\
\hline (1) & (2) & (3) & (4) & (5) & (6) \\
\hline $\begin{array}{c}\text { Variable } \\
\text { Independiente }\end{array}$ & Caso & Estadístico F & Decisión $\mathrm{H}_{0}$ & $\begin{array}{l}\text { Coeficiente de } \\
\text { Corrección del } \\
\text { Error (EC) }\end{array}$ & $\begin{array}{c}\text { Valor p Coeficiente } \\
\text { a Largo Plazo }\end{array}$ \\
\hline IPC & IV & 4.06 & Aceptar $\mathrm{H}_{0}$ & --- & --- \\
\hline $\mathrm{K} / \mathrm{PIB}$ & IV & 3.10 & Aceptar $\mathrm{H}_{0}$ & --- & --- \\
\hline $\mathrm{O} / \mathrm{K}$ & IV & 6.43 & Rechazar $\mathrm{H}_{0} * * *$ & $-0.4586^{*}$ & 0.040 \\
\hline $\mathrm{O} / \mathrm{PIB}$ & IV & 5.65 & Rechazar $\mathrm{H}_{0} * * *$ & $-0.4442 *$ & 0.031 \\
\hline K & III & 4.53 & Aceptar $\mathrm{H}_{0}$ & --- & --- \\
\hline $\mathrm{O}$ & IV & 5.82 & Rechazar $\mathrm{H}_{0}^{* * *}$ & $-0.4371 *$ & 0.024 \\
\hline
\end{tabular}

H0: No existe relación a largo plazo entre las variables.
* Significativo al $1 \%$
** Significativo al $2.5 \%$
*** Significativo al $5 \%$
**** Significativo al 10\%

Fuente: Elaboración propia.

De acuerdo con los hallazgos que aparecen en la Tabla 4 no es posible rechazar la hipótesis de que no existe cointegración entre el IPC y los dos indicadores del crecimiento económico, el $P I$ y el PIB. Tampoco se puede rechazar esa hipótesis en el caso del PIB y los indicadores que involucran de alguna manera a la capitalización del mercado. Los resultados que se muestran en la Tabla 5 indican que tampoco hay cointegración entre los logaritmos de la capitalización del mercado y del volumen de operaciones con el logaritmo del PIB. 
Tabla 5

Resultados de las pruebas de límites: Logaritmos de las variables

Panel A. Variable de Respuesta: $\ln (\mathrm{PI})$

Datos Mensuales

\begin{tabular}{cccccc}
\hline & \multicolumn{5}{c}{ Resultados ARDL } \\
\cline { 2 - 4 } \begin{tabular}{c} 
Variable \\
\cline { 2 - 5 } Independiente
\end{tabular} & Caso & Estadístico $F$ & Decisión $\mathrm{H}_{0}$ & $\begin{array}{c}\text { Coeficiente de } \\
\text { Corrección del Error }\end{array}$ & $\begin{array}{c}\text { Valor } p \text { Coeficiente } \\
\text { a Largo Plazo }\end{array}$ \\
\hline $\ln (\mathrm{IPC})$ & IV & 9.66 & Rechazar $\mathrm{H}_{0}^{*}$ & -0.1266 & 0.000 \\
$\ln (\mathrm{K})$ & III & 14.30 & Rechazar $\mathrm{H}_{0}^{*}$ & -0.1407 & 0.000 \\
$\ln (\mathrm{O})$ & III & 8.10 & Rechazar $\mathrm{H}_{0}^{*}$ & -0.1102 & 0.003 \\
\hline
\end{tabular}

Panel B. Variable de Respuesta: $\ln (\mathrm{PIB})$

Datos Trimestrales

\begin{tabular}{|c|c|c|c|c|c|}
\hline \multirow[b]{2}{*}{ (1) } & \multicolumn{3}{|c|}{ Resultados ARDL } & \multirow[b]{2}{*}{ (5) } & \multirow[b]{2}{*}{ (6) } \\
\hline & (2) & (3) & (4) & & \\
\hline $\begin{array}{c}\text { Variable } \\
\text { Independiente }\end{array}$ & Caso & Estadístico $F$ & Decisión $\mathrm{H}_{0}$ & $\begin{array}{c}\text { Coeficiente de } \\
\text { Corrección del Error }\end{array}$ & $\begin{array}{c}\text { Valor } p \text { Coeficiente } \\
\text { a Largo Plazo }\end{array}$ \\
\hline $\ln (\mathrm{IPC})$ & IV & 6.01 & Rechazar $\mathrm{H}_{0}^{* *}$ & -0.4333 & 0.066 \\
\hline $\ln (\mathrm{K})$ & IV & 4.27 & Aceptar $\mathrm{H}_{0}$ & --- & --- \\
\hline $\ln (\mathrm{O})$ & III & 0.99 & Aceptar $\mathrm{H}_{0}$ & --- & --- \\
\hline
\end{tabular}

H0: No existe relación a largo plazo entre las variables.

* Significativo al $1 \%$

*** Significativo al $5 \%$

Fuente: Elaboración propia.

Los hallazgos sobre la ausencia de una relación a largo plazo entre el IPC y los indicadores del crecimiento económico es inesperada, ya que estudios previos realizados en México encontraron una relación de largo plazo del IPC con el PI y el PIB. Además, a pesar de que el $P I B$, el $P I$ y el $P I B$ son estacionarias en primeras diferencias, no existe una combinación que genere un nuevo proceso estacionario entre el IPC y cualesquiera las dos variables asociadas con el crecimiento económico. Este hallazgo significa que no es posible realizar una verificación cruzada de causalidad como la que sugieren las teorías de interdependencia, de impulso de la demanda o de anticipación a la oferta, por ejemplo ${ }^{7}$.

\footnotetext{
${ }^{7}$ Según lo que plantean Castillo-Ponce et al. (2015), este resultado también podría interpretarse como evidencia en contra de la Hipótesis de los Mercados Eficientes.
} 
Tabla 6

Resultados de las pruebas de no causalidad de Granger: Variables en niveles

Panel A: Variable de Respuesta ( $Y$ ): PI

Datos Mensuales

\begin{tabular}{|c|c|c|c|c|c|}
\hline \multirow[b]{3}{*}{$\begin{array}{c}\text { Variable } \\
\text { Explicativa }(X)\end{array}$} & (2) & (3) & (4) & (5) & (6) \\
\hline & \multicolumn{2}{|c|}{$X$ No Causa a $Y$} & \multicolumn{2}{|c|}{$Y$ No Causa a $X$} & \multirow[b]{2}{*}{$\begin{array}{c}\text { Dirección de } \\
\text { Causalidad }\end{array}$} \\
\hline & Valor $p$ & Decisión $\mathrm{H}_{0}$ & Valor $p$ & Decisión $\mathrm{H}_{0}$ & \\
\hline IPC & 0.060 & Rechazar $\mathrm{H}_{0}$ *** & 0.376 & Aceptar $\mathrm{H}_{0}$ & $\mathrm{PI} \leftarrow \mathrm{IPC}$ \\
\hline $\mathrm{K} / \mathrm{PI}$ & 0.009 & Rechazar $\mathrm{H}_{0} *$ & 0.057 & Rechazar $\mathrm{H}_{0} * *$ & $\mathrm{PI} \leftrightarrow \mathrm{K} / \mathrm{PI}$ \\
\hline $\mathrm{O} / \mathrm{K}$ & 0.175 & Aceptar $\mathrm{H}_{0}$ & 0.465 & Aceptar $\mathrm{H}_{0}$ & Ninguna \\
\hline $\mathrm{O} / \mathrm{PI}$ & 0.615 & Aceptar $\mathrm{H}_{0}$ & 0.777 & Aceptar $\mathrm{H}_{0}$ & Ninguna \\
\hline K & 0.019 & Rechazar $\mathrm{H}_{0} * *$ & 0.365 & Aceptar $\mathrm{H}_{0}$ & $\mathrm{PI} \leftarrow \mathrm{K}$ \\
\hline $\mathrm{O}$ & 0.636 & Aceptar $\mathrm{H}_{0}$ & 0.802 & Aceptar $\mathrm{H}_{0}$ & Ninguna \\
\hline
\end{tabular}

Panel B: Variable de Respuesta (Y): PIB

Datos Trimestrales

(1)

Variable
Explicativa $(X)$

IPC

$\mathrm{O} / \mathrm{K}$
(2)

(3)

$X$ No Causa a $Y$

\begin{tabular}{cc}
$X$ No Causa a $Y$ \\
\hline Valor $p$ & Decisión $\mathrm{H}_{0}$ \\
0.011 & Rechazar $\mathrm{H}_{0} * *$ \\
0.418 & Aceptar $\mathrm{H}_{0}$
\end{tabular}

H0: No existe relación de causalidad.

* Significativo al $1 \% \quad * * *$ Significativo al $10 \%$

** Significativo al 5\%

Fuente: Elaboración propia.

En los modelos en los que se rechazó la hipótesis de no cointegración, los coeficientes del término de corrección del error (ECT) resultaron negativos y muy significativos, por lo que se confirma la relación a largo plazo entre las variables. Además, de acuerdo con estos resultados, entre el 8 y el $10 \%$ del desequilibrio entre el PI y otras variables se corrige en el periodo de un mes, excepto en el caso de la relación entre el PI y la capitalización del mercado, en donde la corrección es más lenta. Específicamente, son necesarios entre siete y diez meses para corregir la mitad del desequilibrio. Para los modelos que involucran a $\ln (P I)$ entre el 11 


\section{J. D. Villarreal Samaniego / Contaduría y Administración 66(3), 2021, 1-23 \\ http://dx.doi.org/10.22201/fca.24488410e.2021.2259}

y el $14 \%$ del desequilibrio se corrige a lo largo de un mes. En el caso modelos que involucran al $P I B$ se advierte que la mitad del desequilibrio persiste solamente por un poco más de un trimestre, lo que también ocurre cuando se considera la relación entre $\ln (P I B)$ y $\ln (I P C)$.

\section{Pruebas de No Causalidad de Granger}

En las Tablas 6 y 7 aparecen los resultados de las pruebas de no causalidad en el sentido de Granger mediante el procedimiento de Toda \& Yamamoto (1995) ${ }^{8}$. Según se muestra en el panel A de la Tabla 6, tanto el IPC como la capitalización del mercado causan a PI, aunque en el primer caso la relación resultó significativa únicamente al 10\%. También se observa que hay una causalidad bidireccional significativa entre el $P I$ la razón $K / P I$. Sin embargo, no se encontró evidencia de causalidad entre el PI con alguna de los indicadores que involucran al volumen de operaciones. Los resultados mostrados en el panel B también indican causalidad significativa del $I P C$ hacia el $P I B$, así como del $P I B$ hacia la razón de rotación del mercado.

En el panel A de la Tabla 7 se observa una causalidad bidireccional entre los cambios porcentuales del PI con los del IPC y los de la capitalización del mercado. También se encontró una relación causal muy significativa del cambio porcentual de $P I$ hacia el cambio porcentual en el volumen de las operaciones en el mercado. Los resultados reportados en el panel B indican que los cambios porcentuales tanto del IPC, los de la capitalización del mercado y los del volumen de operaciones causan los cambios porcentuales del $P I B$, aunque solamente a un nivel de significancia del $10 \%$ en este último caso. En términos generales, los resultados favorecen el punto de vista de la teoría de la anticipación a la oferta. Sin embargo, también hay cierta evidencia a favor de los puntos de vista de la interdependencia y del impulso de la demanda.

\section{Conclusiones}

El presente estudio contribuye al análisis de la relación a largo plazo y causal entre el desarrollo del mercado accionario y el crecimiento económico, enfocándose de forma específica en el caso de México, el segundo país emergente más importante de Latinoamérica en términos del producto interno bruto. Además de la incorporación de cambios estructurales a través de variables binarias, la contribución se hace aplicando métodos eficaces, que no se han utilizado en estudios previamente realizados para la economía mexicana. Específicamente, para determinar la relación a largo plazo de las variables, en esta investigación se emplea el modelo

\footnotetext{
${ }^{8}$ En resultados no reportados por razones de brevedad se encontró que las raíces inversas de la ecuación característica presentaron estabilidad dinámica al mantenerse dentro del círculo unitario, por lo que los modelos VAR fueron estacionarios casi en todos los casos. Sin embargo, los modelos que relacionan al $P I B$ con $O$ y $K$, así como con las razones $O / P I B$ y $K / P I B$ resultaron dinámicamente inestables por lo que no fueron considerados para análisis subsecuentes.
} 
ARDL con prueba de límites propuesto por Pesaran et al. (2001) en tanto que para probar la no causalidad en el sentido de Granger se usa el modelo de Toda \& Yamamoto (1995).

Tabla 7

Resultados de las pruebas de no causalidad de Granger: Logaritmos de las variables

Panel A: Variable de Respuesta $(Y): \ln (\mathrm{PI})$

Datos Mensuales

(1)

(2)

(3)

(4)

(5)

(6)

\begin{tabular}{cccccc} 
& \multicolumn{2}{c}{$X$ No Causa a $Y$} & \multicolumn{2}{c}{$Y$ No Causa a $X$} & Dirección de \\
\cline { 2 - 4 } $\begin{array}{c}\text { Variable } \\
\text { Explicativa }(X)\end{array}$ & Valor $p$ & Decisión $\mathrm{H}_{0}$ & Valor $p$ & Decisión $\mathrm{H}_{0}$ & Causalidad \\
\hline $\ln (\mathrm{IPC})$ & 0.000 & Rechazar $\mathrm{H}_{0} *$ & 0.027 & Rechazar $\mathrm{H}_{0} * *$ & $\ln (\mathrm{PI}) \leftrightarrow \ln (\mathrm{IPC})$ \\
$\ln (\mathrm{K})$ & 0.001 & Rechazar $\mathrm{H}_{0} *$ & 0.063 & Rechazar $\mathrm{H}_{0} * * *$ & $\ln (\mathrm{PI}) \leftrightarrow \ln (\mathrm{K})$ \\
$\ln (\mathrm{O})$ & 0.400 & Aceptar $\mathrm{H}_{0}$ & 0.004 & Rechazar $\mathrm{H}_{0} *$ & $\ln (\mathrm{PI}) \rightarrow \ln (\mathrm{O})$ \\
\hline
\end{tabular}

Panel B: Variable de Respuesta (Y): PIB

Datos Trimestrales
(1)
(2)
(3)
(4)
(5)
(6)

\begin{tabular}{|c|c|c|c|c|c|}
\hline \multirow[b]{2}{*}{$\begin{array}{c}\text { Variable } \\
\text { Explicativa }(X)\end{array}$} & \multicolumn{2}{|c|}{$X$ No Causa a $Y$} & \multicolumn{2}{|c|}{$Y$ No Causa a $X$} & \multirow[b]{2}{*}{$\begin{array}{c}\text { Dirección de } \\
\text { Causalidad }\end{array}$} \\
\hline & Valor $p$ & Decisión $\mathrm{H}_{0}$ & Valor $p$ & Decisión $H_{0}$ & \\
\hline $\ln (\mathrm{IPC})$ & 0.000 & Rechazar $\mathrm{H}_{0} *$ & 0.776 & Aceptar $\mathrm{H}_{0}$ & $\ln (\mathrm{PIB}) \leftarrow \ln (\mathrm{IPC})$ \\
\hline $\ln (\mathrm{K})$ & 0.000 & Rechazar $\mathrm{H}_{0} *$ & 0.921 & Aceptar $\mathrm{H}_{0}$ & $\ln (\mathrm{PIB}) \leftarrow \ln (\mathrm{K})$ \\
\hline $\ln (\mathrm{O})$ & 0.088 & Rechazar $\mathrm{H}_{0} * * *$ & 0.330 & Aceptar $\mathrm{H}_{0}$ & $\ln (\mathrm{PIB}) \leftarrow \ln (\mathrm{O})$ \\
\hline
\end{tabular}

H0: No existe relación de causalidad.

* Significativo al $1 \% \quad * * *$ Significativo al $10 \%$

** Significativo al $5 \%$

Fuente: Elaboración propia.

Los resultados de las pruebas de raíz unitaria con cambios estructurales resaltan la influencia de los principales episodios de estrés financiero ocurridos desde mediados de la década de los noventa hasta 2013 en las variables bajo análisis. Sin embargo, de acuerdo con los resultados de las pruebas de cambios estructurales determinados secuencialmente de Bai \& Perron (2003), el shock de la crisis mexicana de mediados de la década de 1990 fue el más relevante, ya que impactó a todas las variables examinadas. Por otra parte, de acuerdo con los resultados el shock de la Gran Recesión de 2008-2009 fue relativamente limitado en México, ya que únicamente involucró al IPC y al volumen monetario de las operaciones del mercado accionario. Muy posiblemente esto se deba a que la economía mexicana tenía en fundamentos sólidos que le permitieron mitigar el impacto de esa crisis. 
El episodio denominado Rabieta de Disminución Gradual (Taper Tantrum) de 2013 tuvo efectos importantes sobre la rotación del mercado accionario. Es muy probable que, ante el posible incremento de las tasas de interés en Estados Unidos, se diera una huida hacia la calidad (flight to quality), incrementando considerablemente el volumen de operaciones y reduciendo, aunque relativamente menos, la capitalización del mercado en México. Estas condiciones no se presentaron durante la crisis financiera de 2008-2009, por lo que la rotación del mercado se vio comparativamente menos afectada por ese episodio.

Los resultados de los modelos ARDL con prueba de límites indican que existe una relación a largo plazo entre las variables del crecimiento económico con la mayoría de las variables relativas al desarrollo del mercado accionario. Además, los resultados de los coeficientes del término de corrección del error confirmaron la cointegración de las variables. Estos hallazgos coinciden, en términos generales, con los reportados por Castillo-Ponce et al. (2015) y Ron Delgado (2001) para la economía y el mercado accionario mexicanos. No obstante, una discrepancia importante con respecto a los resultados de esas investigaciones es que en este estudio no se encontró evidencia de relación a largo plazo entre la dinámica del IPC y la de los indicadores de crecimiento económico. Se considera que esta divergencia se puede deber a los métodos empleados, a la incorporación de los cambios estructurales dentro de los modelos realizada en este estudio, y a las diferencias en los periodos examinados.

Varios de los hallazgos de las pruebas de no causalidad de Granger favorecen al punto de vista de teoría de la anticipación a la oferta, puesto que se encontró una relación de causalidad significativa de las variables del mercado accionario hacia las del crecimiento económico. Estos resultados coinciden con los de otros estudios realizados en diversos países (Adebayo et al., 2020; Caporale et al., 2004; Pradhan, 2018), aunque contrastan con los reportados por Pan \& Mishra (2018), quienes encontraron una relación causal de la economía hacia el mercado accionario en China.

Específicamente, se identificó una relación de causalidad de la capitalización del mercado hacia el índice de producción industrial, así como del IPC tanto hacia el PI como hacia el $P I B$. Estos resultados pueden estar relacionados con la apertura comercial y financiera de México desde mediados de la década de 1990. Cabe señalar, sin embargo, que el hallazgo de que no existe relación a largo plazo entre el IPC y las variables del crecimiento económico no permite la verificación cruzada de estos resultados de causalidad, lo que abre la posibilidad para realizar futuras investigaciones sobre este aspecto. Por otra parte, los resultados obtenidos indican que la capitalización del mercado causa en el sentido de Granger el crecimiento económico medido a través del $P I$.

También se identificó una relación bidireccional de causalidad del PI y de la profundización del mercado medida a través de la razón $K / P I$, lo cual es particularmente relevante para un país emergente de acuerdo con el punto de vista de la interdependencia entre el crecimiento 
económico y el desarrollo del mercado accionario. Por último se descubrió que hay una relación de causalidad del PIB hacia la liquidez del mercado, medida con la razón de volumen de operaciones a capitalización total del mercado, por lo que se concluye que el crecimiento económico influye sobre la eficiencia de operación del mercado accionario.

Los resultados de este trabajo de investigación tienen diversas implicaciones para los responsables de formular políticas públicas. En primer lugar, sobre la base de la relación de causalidad del mercado accionario hacia la economía en México, se debe fomentar la estabilidad, la liquidez y la transparencia de dicho mercado y proteger a los inversionistas que ahí participan. En segundo término, deben implementarse políticas que, dentro de un marco regulatorio que considere los elementos mencionados, también faciliten el acceso de las empresas a los recursos que se pueden obtener a través del mercado accionario. Finalmente, se deben establecer los mecanismos para facilitar la entrada de una base más amplia de inversionistas a ese mercado, de modo que sus recursos se canalicen eficientemente hacia la economía real y se promueva su crecimiento.

Uno de los temas que queda abierto para futuras investigaciones es el de la aceptación de la hipótesis de no causalidad a pesar de la relación a largo plazo identificada entre el índice de producción industrial y el volumen de operaciones. Un camino para explorar sería la posibilidad de que este resultado se relacionara con variables omitidas que pudieran afectar tanto al volumen de operaciones como al PI (Lütkepohl, 1982). Concretamente, los modelos evaluados aquí y que consideran a esas variables, podrían incorporar simultáneamente otras como, por ejemplo, la capitalización del mercado. Un aspecto que también podría abordarse en estudios posteriores es el relacionado con la influencia que tienen el mercado de bonos o el sistema bancario, sobre el crecimiento de la economía. Finalmente, un tema que valdría la pena considerar para profundizar en su estudio es relativo al desarrollo del mercado accionario en México y su contribución a la reducción de los costos de financiamiento para las empresas, así como el impacto que esto pudiera tener sobre el crecimiento de la economía.

\section{Referencias}

Aali-Bujari, A., Venegas-Martínez, F., \& Pérez-Lechuga, G. (2017). Impact of the Stock Market Capitalization and the Banking Spread in Growth and Development in Latin America: A Panel Data Estimation with System GMM. Contaduría y Administración, 62(5), 1427-1441. https://doi.org/10.1016/J.CYA.2017.09.005

Adebayo, T. S., Awosusi, A. A., \& Eminer, F. (2020). Stock Market-Growth Relationship in an Emerging Economy: Empirical Finding from ARDL-Based Bounds and Causality Approaches. Journal of Economics and Business, 3(2), 903-916. https://doi.org/10.31014/aior.1992.03.02.247

Bai, J., \& Perron, P. (2003). Computation and Analysis of Multiple Structural Change Models. Journal of Applied Econometrics, 18(1), 1-22. https://doi.org/10.1002/jae.659

Beck, T., Demirgüç-Kunt, A., \& Levine, R. (2010). Financial Institutions and Markets Across Countries and Over Time: The Updated Financial Development and Structure Database. The World Bank Economic Review, 24(1), 77-92. https://doi.org/10.1093/wber/lhp016 


\section{J. D. Villarreal Samaniego / Contaduría y Administración 66(3), 2021, 1-23 \\ http://dx.doi.org/10.22201/fca.24488410e.2021.2259}

Bhatt, V. V. (1995). Financial Systems, Innovation, and Development. New Delhi: Sage.

Blum, D., Federmair, K., Fink, G., \& Haiss, P. (2002). The Financial-Real Sector Nexus: Theory and Empirical Evidence (No. 43). Vienna.

Boubakari, A., \& Jin, D. (2010). The Role of Stock Market Development in Economic Growth: Evidence from Some Euronext Countries. International Journal of Financial Research, 1(1), 14-20. https://doi.org/10.5430/ ijfr.v1n1p14

Boyd, J., \& Smith, B. (1996). The Coevolution of the Real and Financial Sectors in the Growth Process. The World Bank Economic Review, 10(2), 371-396. https://doi.org/10.1093/wber/10.2.371

Brown, R. L., Durbin, J., \& Evans, J. M. (1975). Techniques for Testing the Constancy of Regression Relationships over Time. Journal of the Royal Statistical Society. Series B (Methodological), 37(2), 149-192. https://doi. org/10.2307/2984889

Caporale, G. M., Howells, P. G., \& Soliman, A. M. (2004). Stock Market Development and Economic Growth: The Causal Linkage. Journal of Economic Development, 29(1), 33-50. https://doi.org/10.1109/TIP.2011.2118224

Castillo-Ponce, R. A., Rodriguez-Espinosa, M. de L., \& Gaytan-Alfaro, E. D. (2015). Stock Market Development and Economic Performance: The Case of Mexico. Revista de Análisis Económico, 30(1), 41-56. https://doi. org $110.4067 / \mathrm{S} 0718-88702015000100003$

Cole, D. C., \& Shaw, E. S. (1974). Financial Deepening in Economic Development. The Journal of Finance, 29(4), 1345. https://doi.org/10.2307/2978421

Cuche, N. A., \& Hess, M. K. (1999). A Monthly Series of Swiss GDP: Business Cycle Indicator and Research Tool. Retrieved June 22, 2018, from http://id3414.securedata.net/cuche/download/pdf/papers/wp-0899.pdf

Engle, R. F., \& Granger, C. W. J. (1987). Co-Integration and Error Correction: Representation, Estimation, and Testing. Econometrica, 55(2), 251-276. https://doi.org/10.2307/1913236

Fink, G., Haiss, P., \& Vukšić, G. (2009). Contribution of Financial Market Segments at Different Stages of Development: Transition, Cohesion and Mature Economies Compared. Journal of Financial Stability, 5(4), 431-455. https://doi.org/10.1016/j.jfs.2008.05.002

Ghimire, B., \& Giorgioni, G. (2013). Puzzles in the Relationship Between Financial Development and Economic Growth. Journal of Applied Finance \& Banking, 3(5), 199-222. Retrieved September 7, 2018, from http:// www.scienpress.com/Upload/JAFB/Vol 3_5_15.pdf

Goldsmith, R. W. (1959). Financial Structure and Development. In R. W. Goldsmith (Ed.), The Comparative Study of Economic Growth and Structure (pp. 114-123). National Bureau of Economic Research.

Graff, M. A. (2001). Financial Development and Economic Growth - A New Empirical Analysis. SSRN Electronic Journal. https://doi.org/10.2139/ssrn.258928

Hatemi-J, A. (2008). Tests for Cointegration with Two Unknown Regime Shifts with an Application to Financial Market Integration. Empirical Economics, 35(3), 497-505. https://doi.org/10.1007/s00181-007-0175-9

Ho, S. Y., \& Iyke, B. N. (2017). On the Causal Links Between the Stock Market and the Economy of Hong Kong. Contemporary Economics, 11(3), 343-362. https://doi.org/10.5709/ce.1897-9254.247

Hoque, M. E., \& Yakob, N. A. (2017). Revisiting Stock Market Development and Economic Growth Nexus: The Moderating Role of Foreign Capital Inflows and Exchange Rates. Cogent Economics and Finance, 5(1-17). https://doi.org/10.1080/23322039.2017.1329975

Inusah, N. (2018). Toda-Yamamoto Granger No-causality Analysis of Stock Market Growth and Economic Growth in Ghana. Journal of Accounting, Business and Finance Research, 3(1), 36-46. https://doi. org/10.20448/2002.31.36.46

Johansen, S. (1988). Statistical Analysis of Cointegration Vectors. Journal of Economic Dynamics and Control, 12(2), 231-254. https://doi.org/10.1016/0165-1889(88)90041-3

Johansen, S. (1991). Estimation and Hypothesis Testing of Cointegration Vectors in Gaussian Vector Autoregressive Models. Econometrica, 59(6), 1551. https://doi.org/10.2307/2938278

Kajurová, V. (2017). A Note on Relationship Between Economic Activity and Stock Market Development: A Case of Euro Area Countries. Acta Universitatis Agriculturae et Silviculturae Mendelianae Brunensis, 65(6), 19531965. https://doi.org/10.11118/actaun201765061953 


\section{J. D. Villarreal Samaniego / Contaduría y Administración 66(3), 2021, 1-23 \\ http://dx.doi.org/10.22201/fca.24488410e.2021.2259}

Kassimatis, K., \& Spyrou, S. I. (2001). Stock and Credit Market Expansion and Economic Development in Emerging Markets: Further Evidence Utilizing Cointegration Analysis. Applied Economics, 33(8), 1057-1064. https://doi.org/10.1080/000368401300182824

King, R. G., \& Levine, R. (1993). Finance and Growth: Schumpeter Might Be Right. The Quarterly Journal of Economics, 108(3), 717-737. https://doi.org/10.2307/2118406

Lahura, E., \& Vega, M. (2017). Stock Market Development and Real Economic Activity in Peru. Empirical Economics, 53(3), 1011-1038. https://doi.org/10.1007/s00181-016-1149-6

Laurencenson, J., \& Chai, J. C. H. (2003). Financial Reform and Economic Development in China. Northpampton, MA: Edward Elgar Publishing Limited.

Levine, R., \& Zervos, S. (1998). Stock Markets, Banks, and Economic Growth. The American Economic Review, 88(3), 537-558. https://doi.org/10.1016/j.ribaf.2006.05.002

Lucas, R. E. (1988). On the Mechanics of Economic Development. Journal of Monetary Economics, 22(1), 3-42. https://doi.org/10.1016/0304-3932(88)90168-7

Lütkepohl, H. (1982). Non-causality Due to Omitted Variables. Journal of Econometrics, 19(2-3), 367-378. https://doi.org/10.1016/0304-4076(82)90011-2

McKinnon, R. I. (1973). Money and Capital in Economic Development. Washington, D.C.: Brookings Institution Press.

Men, M., \& Li, R. (2006). An Empirical Study on the Relationship Between Stock Index and the National Economy: The Case of China. In University of International Business \& Economics Working Paper. Retrieved August 30, 2018, from https://faculty.washington.edu/karyiu/confer/beijing06/papers/men-li.pdf

Menegaki, A. N. (2019). The ARDL Method in the Energy-Growth Nexus Field; Best Implementation Strategies. Economies, 7(4), 105. https://doi.org/10.3390/economies7040105

Mishkin, F. S., \& Eakins, S. G. (2015). Financial Markets and Institutions. Reading, Mass: Addison Wesley.

Newey, W. K., \& West, K. D. (1987). A Simple, Positive Semi-Definite, Heteroskedasticity and Autocorrelation Consistent Covariance Matrix. Econometrica, 55(3), 703-708. https://doi.org/10.2307/1913610

Pan, L., \& Mishra, V. (2018). Stock Market Development and Economic Growth: Empirical Evidence from China. Economic Modelling, 68(January), 661-673. https://doi.org/10.1016/j.econmod.2017.07.005

Paramati, S. R., \& Gupta, R. (2013). An Empirical Analysis of Stock Market Performance and Economic Growth: Evidence from India. SSRN Electronic Journal. https://doi.org/10.2139/ssrn.2335996

Patrick, H. T. (1966). Financial Development and Economic Growth in Underdeveloped Countries. Economic Development and Cultural Change, 14(2), 174-189. https://doi.org/10.1086/450153

Perron, P. (1989). The Great Crash, the Oil Price Shock, and the Unit Root Hypothesis. Econometrica, 57(6), 1361-1401. https://doi.org/10.2307/1913712

Pesaran, M. H., \& Pesaran, B. (1997). Working with Microfit 4.0: Interactive Econometric Analysis. Oxford, UK: Oxford University Press.

Pesaran, M. H., Shin, Y., \& Smith, R. J. (2001). Bounds Testing Approaches to the Analysis of Level Relationships. Journal of Applied Econometrics, 16(3), 289-326. https://doi.org/10.1002/jae.616

Pradhan, R. P. (2018). Development of Stock Market and Economic Growth: The G-20 Evidence. Eurasian Economic Review, 8(2), 161-181. https://doi.org/10.1007/s40822-018-0094-4

Robinson, J. (1952). The Generalisation of the General Theory. In The Generalisation of the General Theory and other Essays (2nd. Ed., pp. 1-76). https://doi.org/10.1007/978-1-349-16188-1_1

Ron Delgado, F. E. (2001). Ajuste Dinámico y Equilibrio entre la Producción Industrial y la Actividad Bursátil en México. Momento Económico, 118(1), 21-38. Retrieved November 18, 2017, from http://www.revistas.unam. $\mathrm{mx} / \mathrm{index} . \mathrm{php} / \mathrm{rme} / \mathrm{article} / \mathrm{view} / 4291$

Schumpeter, J. A. (1911). The Theory of Economic Development: An Inquiry into Profits, Capital, Credit, Interest, and the Business Cycle. Boston, MA: Harvard University Press.

Shestha, M. B., \& Chowdury, K. (2005). ARDL Modelling Approach to Testing the Financial Liberalisation Hypothesis (No. WP 05-15). Retrieved from http://www.uow.edu.au/commerce/econ/wpapers.html 
Sims, C. A. (1992). Interpreting the Macroeconomic Time Series Facts. European Economic Review, 36(5), 975 1000. https://doi.org/10.1016/0014-2921(92)90041-T

Tinoco, M. A., Torres, V. H., \& Cabezas, D. A. (2011). Stock Market Development, Financial Development and Economic Growth in Southeast Asia and Latin America. XI Seminario Internacional Sobre La Cuenca Del Pacífico, 1-14. Colima, Mexico: Universidad de Colima.

Toda, H. Y., \& Yamamoto, T. (1995). Statistical Inference in Vector Autoregressions with Possibly Integrated Processes. Journal of Econometrics, 66(1-2), 225-250. https://doi.org/10.1016/0304-4076(94)01616-8

Vahid, F., \& Engle, R. F. (1993). Common Trends and Common Cycles. Journal of Applied Econometrics, 8(4), 341-360. https://doi.org/10.2307/2285000 